\title{
Epidemic Characteristics of Histomonosis in Geese Flocks in the Orenburg Region
}

\section{Pavel I. Khristianovsky, Stanislav A. Platonov, and Vladislav V. Belimenko}

Federal Research Centre of Biological Systems and Agrotechnologies RAS, Orenburg, Russia ORCID:

Pavel I. Khristianovsky: http://orcid.org/0000-0003-3902-4379

\section{Abstract}

In May-June 2019, there was a mortality surge among adult geese on the IP Samodurov farm (in the village of Krasnokholm, Orenburg Region). The deaths stopped as soon as the geese were out of lay. Pathological and microbiological studies established three parasitic diseases in the flock: heterakidosis, histomonosis, and eimeriosis. A one-off seasonal and age-related manifestation of these diseases is due to a reduced body resistance in the winter, huddling behaviour, and a high load in female geese during the laying season.

Corresponding Author: Pavel I. Khristianovsky Paor1953@bk.ru

Published: 5 April 2021 Publishing services provided by Knowledge E

(c) Pavel I. Khristianovsky et al. This article is distributed under the terms of the Commons Attribution License, which permits unrestricted use and redistribution provided that the original author and source are credited.

Selection and Peer-review under the responsibility of the DonAgro Conference Committee.

\section{Introduction}

Histomonosis (blackhead disease) is an invasive disease of poultry due to parasitic infection of a protozoan, Histomonas meleagridis. The main symptoms of histomoniasis are hepatic necrosis, increased cecum, and sulphur-yellow diarrhea [1]. The parasite falls within the order Trichomonadida that is fairly resistant to environmental conditions - it dies within a few hours outside the host [2]. The protozoan parasite is transmitted through the eggs of Heterakis gallinarum, which protect it from environmental impact [3]. Chickens, mainly young birds, get ill; adults can fall ill in the fall and winter, in a huddling environment. $\mathrm{H}$. meleagridis is extremely dangerous for turkeys; mortality rate can reach $100 \%[4,5]$. Histomonosis in goose flocks is rarely recorded in the Russian Federation; it is clinically manifested in goslings in the autumn-winter period $[6,7]$.

Although the disease has been known for over a hundred years with some effective therapies and preventions to be in, the disease is a current huge challenge in the poultry industry. This is due to a negative effect that the pharmaceuticals employed to combat them can have on the quality of products, and in some countries such drugs are simply prohibited. In this context, in recent years there has been an excess incidence among galliformes around the world [8-10]. 
There is currently no effective drug approved for use in poultry production in the United States [11]. Plant-based medicines are being developed, though, that can effectively diminish a harmful action of protozoans towards the gastrointestinal tract and overall productivity of poultry [12], including uninfected birds [13, 14].

In May-June 2019, an enzootic outbreak of an unclear etiology in adult geese occurred on IP Samodurov farm (the village of Krasnokholm, Orenburg Region). Female geese fell ill at the end of lay. There were some clinical signs, including depression, reduced appetite, listlessness, etc. Diarrhoea began in 2-3 days, the birds quickly lost weight. Clinical signs were observed within 3-5 weeks, followed by recovery. Mortality rate was $48 \%$ of the number of infected birds. Post-mortem examination reported, inter alia, peritoneal extraction, accumulations of curd mass in the rectum, grain-sized lesions in the kidneys, congestion in the liver, small lesions in the parenchyma. Helminths were found to be present in the ceca. Identification of specific clinical and pathological signs suggested the presence of histomonosis in geese flocks reared on the farm. In this regard, there was a need to determine the cause of the disease and take preventive measures before the start of the laying season.

\section{Methods and Equipment}

IP Samodurov goose farm is located in the village of Krasnokholm, Orenburg Region. The number of geese reaches 10-12 thousand Large Grey fowls. The flock is kept without water, in poultry houses and aviaries, rate of stocking is high. Household poultry production involves a breeding stock to lay eggs in February - June, followed by incubation period in a household incubator. Forage includes grain mix, plenty of water.

The poultry farm was established in 2015 . Since then, the owner has not disinfested the premises and walking areas. He used formalin vapor disinfection alone. The method is known to fail to destruct an invasive habit of the soil in poultry houses. Consequently, during this period, helminth eggs, protozoa oocysts, and earthworms (reservoir hosts of parasites), inevitably accumulate inside the poultry house and on walking yards.

In addition, it is of great epizootological significance that synanthropic birds (pigeons, sparrows) invading feed and territory can freely get to the walking grounds. The farm is located near the floodplain of the Ural. Therefore, indirect contact with wild waterfowl susceptible to the same diseases as domestic geese is possible.

IP Samodurov goose farm is located in the village of Krasnokholm, Orenburg Region. The number of geese reaches 10-12 thousand Large Grey fowls. The flock is kept 


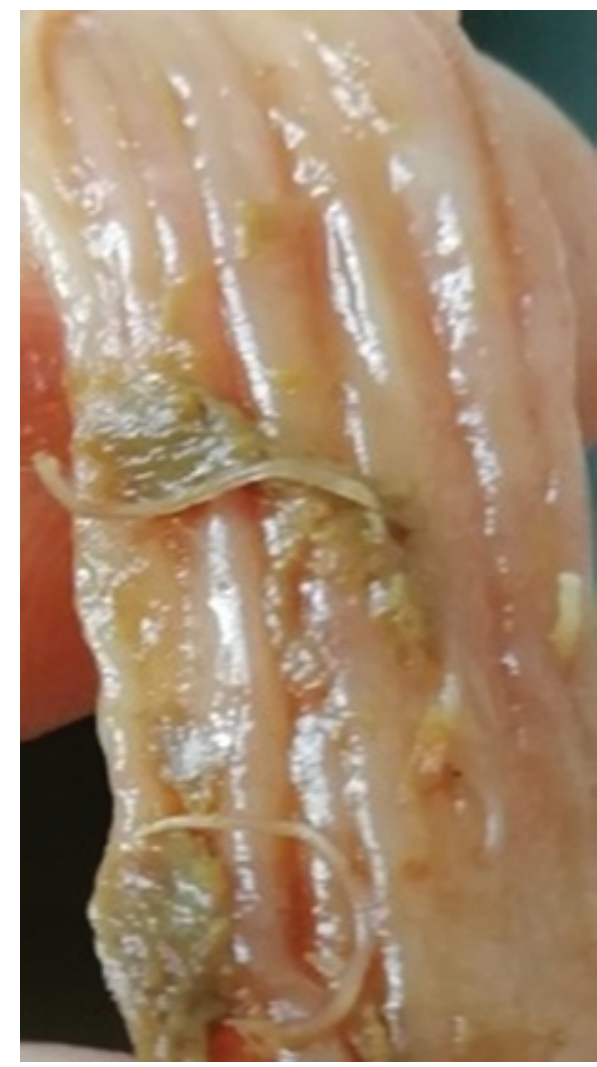

Figure 1: Heterakis gallinarum

without water, in poultry houses and aviaries, rate of stocking is high. Household poultry production involves a breeding stock to lay eggs in February - June, followed by incubation period in a household incubator. Forage includes grain mix, plenty of water.

The poultry farm was established in 2015 . Since then, the owner has not disinfested the premises and walking areas. He used formalin vapor disinfection alone. The method is known to fail to destruct an invasive habit of the soil in poultry houses. Consequently, during this period, helminth eggs, protozoa oocysts, and earthworms (reservoir hosts of parasites), inevitably accumulate inside the poultry house and on walking yards.

In addition, it is of great epizootological significance that synanthropic birds (pigeons, sparrows) invading feed and territory can freely get to the walking grounds. The farm is located near the floodplain of the Ural. Therefore, indirect contact with wild waterfowl susceptible to the same diseases as domestic geese is possible. 


\section{Results}

The study was conducted in the parasitological laboratory of the Department of Microbiology and Infectious Diseases at OSAU on February 11-13, 2020.

\subsection{Helminthovoscopy to investigate faecal matter from geese}

The specimens were collected on 02/10/2020, and delivered to the lab on 02/11/2020. Nine feces specimens were examined using the Fülleborn's method. No helminth eggs were detected. Eimeria oocysts were found to be present in all specimens in an amount of 1 to 10 .

\subsection{Autopsy of a bird}

On $02 / 11 / 2020$, a female goose, aged 2 , was delivered to the laboratory. The goose had been kept in the isolator for two months due to lameness and had low live market quality. The bird was slaughtered for diagnosis in the lab. Post-mortem examination reported a lack of fat and muscle hypotrophy. There were lesions in the liver and purulent necrotic lesions in the kidneys. Heterakis ova were found to be present in the intestinal content. The cecal lining was irritated, 18 helminths Heterakis gallinarum were detected (Fig. 1).

\subsection{Examination of organs}

In the same period, frozen organs of dead geese were delivered to the laboratory (lungs - 6 specimens, kidneys -4 specimens, liver -6 specimens). Once thawed, the organs were examined. The lungs were found to have agonal changes (partial edema), the kidneys - purulo-necrotic lesions (Fig. 2), the liver - passive hepatic congestion and caseation lesions (Fig. 3)

The tissue smears for microscopy were prepared from the kidneys and liver delivered from the household, as well as from the kidneys and liver of the goose subjected to slaughter in the lab. A direct smear method was used to examine kidney tissues. To do this, a caseous mass was taken from a necrotic lesion at the tip of a scalpel, and placed on a glass slide. A drop of isotonic sodium chloride solution was placed nearby, covered with a coverslip and microscopied at low power. Eimeria oocysts Eimeria trichata were found to be present (Fig. 4) in an amount of 5-20 per power field. 


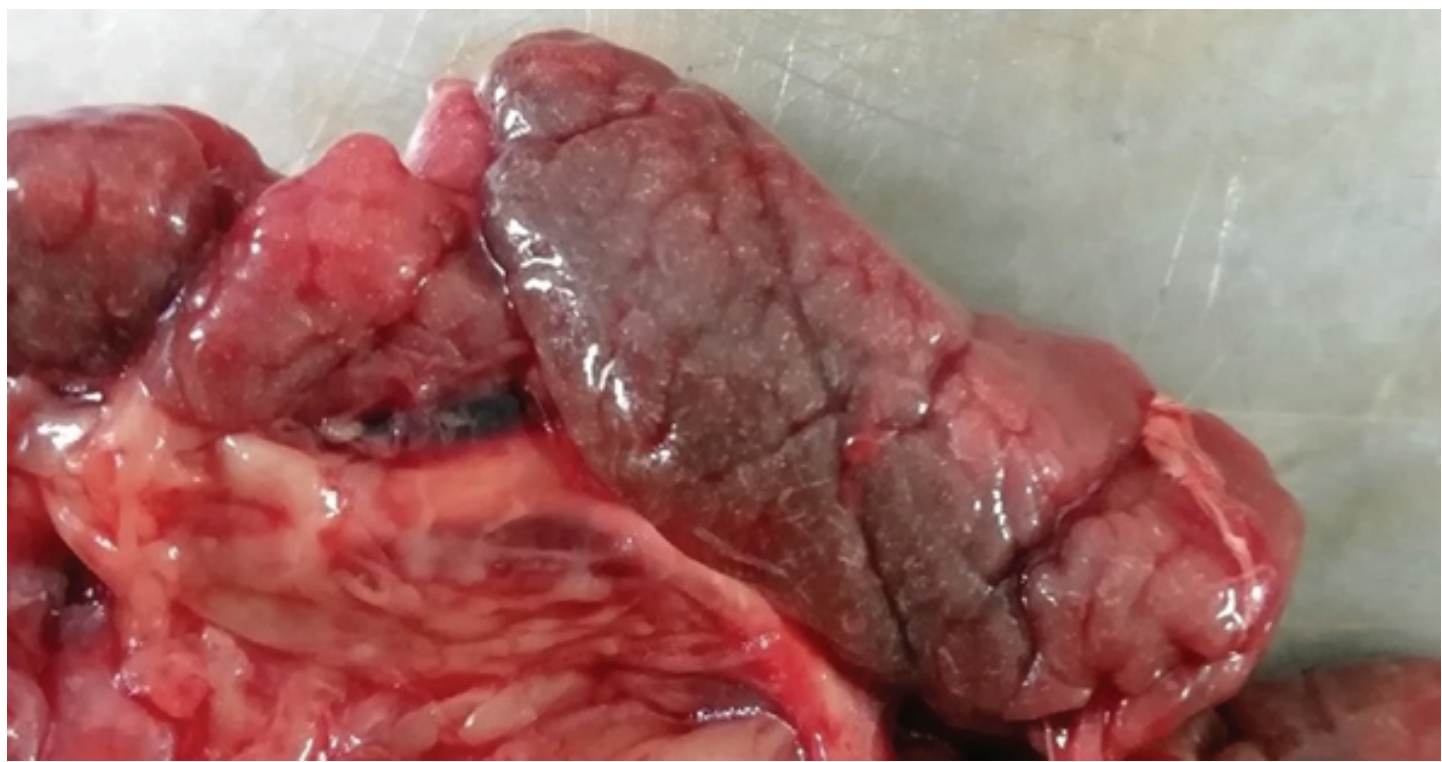

Figure 2: Purulo-necrotic lesions on a kidney

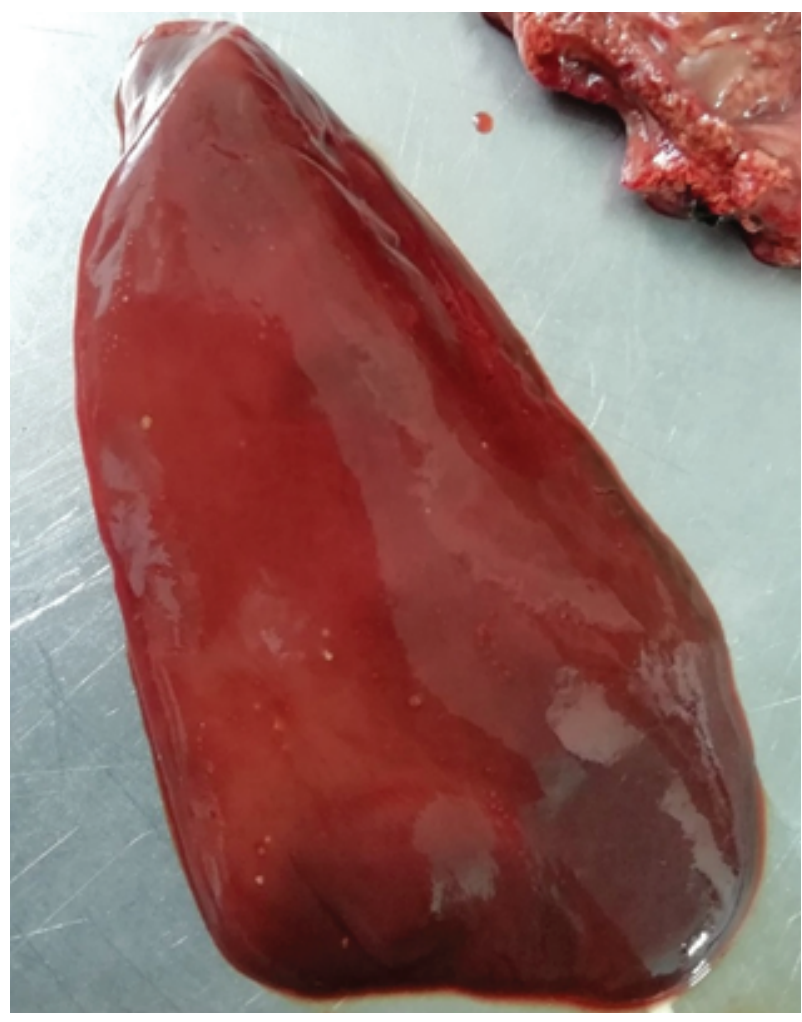

Figure 3: Pathologically changed liver

The liver smears were examined through Romanowsky-Giemsa staining in a microscope immersion system. Amoeba-shaped forms Histomonas meleagridis were found in an amount of 1-10 per power field (Fig. 5).

No blood parasites were found in the blood smears of the goose subjected to slaughter in the lab and examined through Romanowsky-Giemsa staining. 


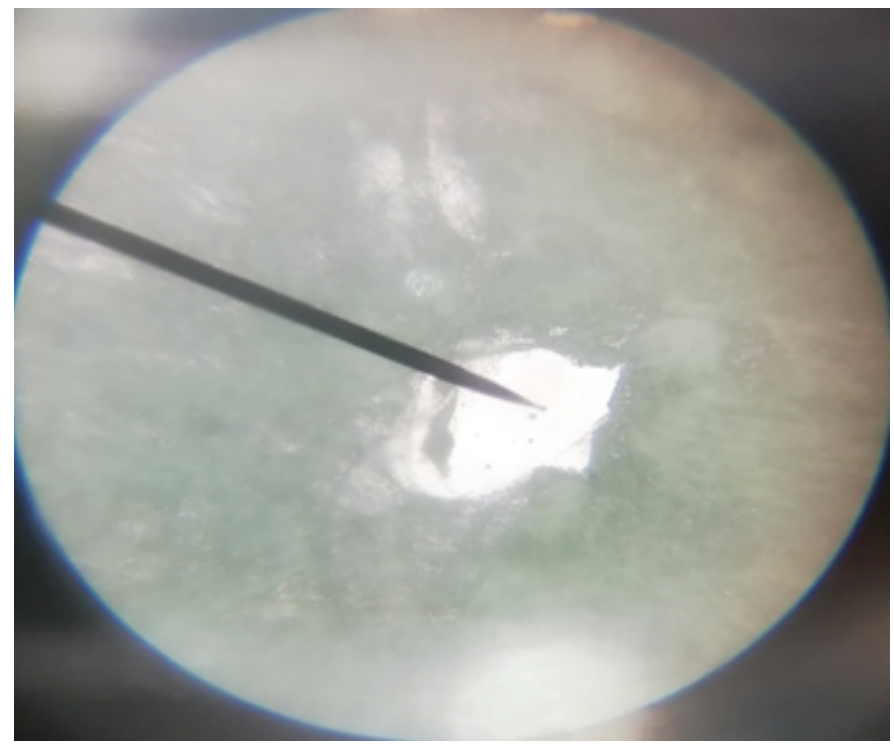

Figure 4: Eimeria truncata in the lumen of uriniferous tubules

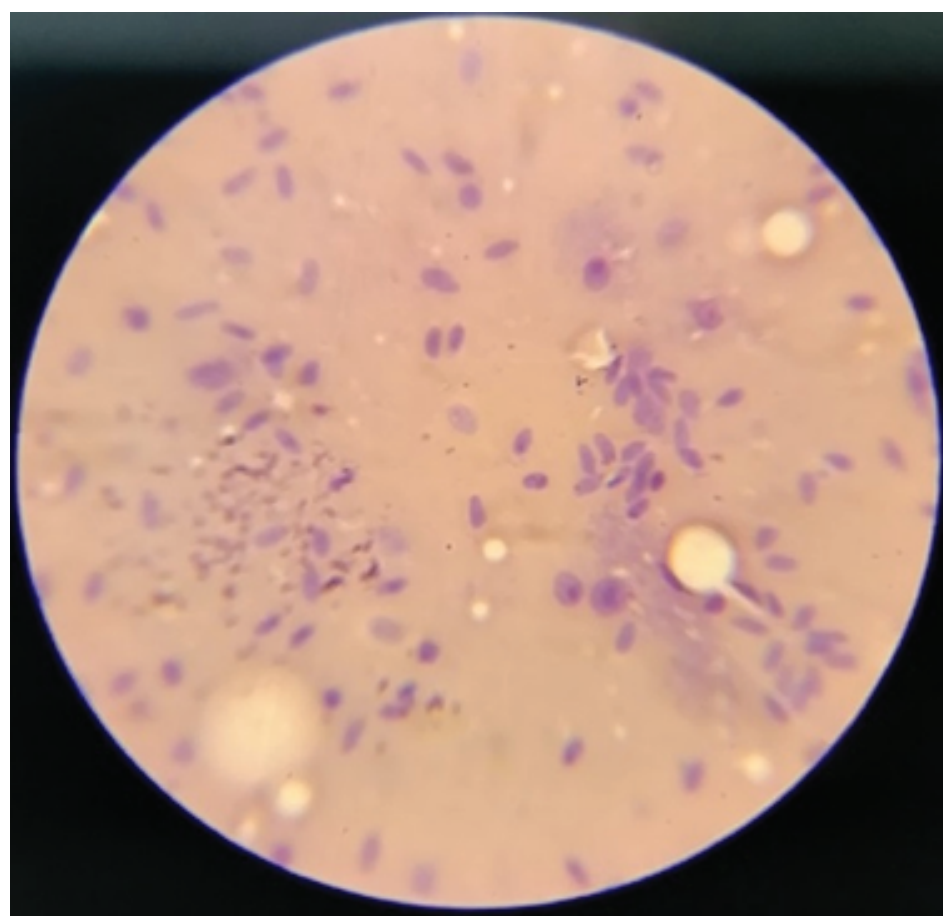

Figure 5: Histomonas meleagridis in a liver smear

\section{Discussion}

According to the literature, geese aged 1-4 months are more susceptible to eimeriosis, usually in summer and autumn. However, seasonality for young chickens in China accounts for April and June [15].

Histomonosis also occurs more often in young flocks under the age of 3 months in the summer-autumn period. Adult geese get sick less frequently, usually in autumn and 
winter. In this case, it was adult geese that were sick during the laying season, i.e., in spring and early summer.

A retrospective study of histomonosis turkey flocks in the USA [16] showed that in all cases the parasite was detected by histopathology. Most cases occurred during the warmer months from April to October, with a clear peak in August. Turkeys in flocks infected with this disease were between the ages of 2 weeks and 15 months, aged 9 weeks on average. In 12 cases, the parasite was found in organs other than the cecum and liver. These include the spleen, kidneys, bursa of Fabricius, lungs, and pancreas. The overall mortality among turkeys from affected flocks ranged from 12 to $65 \%$.

In the said case, a one-off seasonal and age-related manifestation of histomonosis was likely to happen. Apparently, a routinely reduced body resistance in winter was exacerbated by huddling behaviour of the flock. In addition, female geese were weakened by the process of egg laying, which requires large energy and tectonic resources. A factor triggering blackhead invasion and keeping it alive was a helminthic disease heterakidosis, since histomonads can persist in Heterakis ova for a long time.

Heterakis ova are known to carry $\mathrm{H}$. meleagridis, and the co-infection rate in hens is $50.8 \%$ [17].

A reduced body resistance triggered eimeriosis pathogens as well, and the disease turned from a carrier into clinically apparent form.

Moreover, a mortality surge after infection is associated not only with the breed and age of the birds, but also with their feeding style, with different genotypes and levels of virulence of different geographically isolated histomonads [18].

Thus, resulting from the above processes, the target goose-rearing farm was struck by an outbreak of protozoan invasions in geese accompanied by one-off seasonal and age-related characteristics.

\section{Conclusion}

Intestinal and renal eimeriosis, histomonosis, and heterakidosis were found in the geese flocks on the IP Samodurov farm. Currently, these invasions are in a carrier form. The enzootic outbreak of these diseases among the geese was due to a reduced body resistance in winter, huddling behaviour, and a high load on female geese during laying season. 


\section{Acknowledgement}

The study was performed in the framework of the project \#0761-2019-0006.

\section{References}

[1] Liebhart, D., et al. (2017). Histomonosis in Poultry: Previous and Current Strategies for Prevention and Therapy. Avian Pathology, vol. 46, pp. 1-18.

[2] Lotfi, A-R., Abdelwhab, E. and Hafez, H. (2012). Persistence of Histomonas Meleagridis in or on Materials used in Poultry Houses. Avian, vol. 56, pp. 224-6.

[3] Aka, J., et al. (2010). Reoccurrence of Histomonosis in Turkey Breeder Farm. Berliner und Münchener tierärztliche Wochenschrift, vol. 124, pp. 2-7.

[4] Dolka, B., et al. (2015). Histomonosis-An Existing Problem in Chicken Flocks in Poland. Veterinary Research Communications, vol. 39, pp. 189-95.

[5] Nguyen, D. T., et al. (2015). Prevalence and Genetic Characterization of Histomonas Meleagridis in Chickens. Avian Diseases, vol. 59. pp. 309-14.

[6] Osintseva, V. A. and Volkova, E. S. (2007). Study of the Comparative Effectiveness of Drugs for Histomonosis in Geese Flocks Izv. Orenburg State University, vol. 1, issue 13, pp. 71-2.

[7] Chalysheva, E. I. and Safiullin, R. T. (2019). Epizootic Situation for Intestinal Parasitic Protozoa of Young Turkeys on Poultry Farms in Central Russia Theory and Practice of Controlling Parasitic Diseases Russian Academy of Sciences, vol. 20, pp. 690-4.

[8] McDougald, L. R. (2005). Blackhead Disease (Histomoniasis) in Poultry: A Critical Review Avian Diseases, vol. 49, issue 4, pp. 62-76.

[9] Mitra, T., et al. (2018). Unravelling the Immunity of Poultry Against the Extracellular Protozoan Parasite Histomonas meleagridis. Is a Cornerstone for Vaccine Development: A Review Front Immunol, vol. 9, pp. 2518.

[10] Dolka, B., Żbikowski, A. and Dolka, I. (2015). Szeleszczuk Histomonosis - An Existing Problem in Chicken Flocks. Veterinary Research Communications, vol. 39, issue 3, pp. 189-95.

[11] Clark, S. and Kimminau, E. (2017). Critical Review: Future Control of Blackhead Disease (Histomoniasis) in Poultry. Avian Diseases, vol. 61, issue 3, pp. 281-8.

[12] Duffy, C. F., Sims, M. D. and Power, R. F. (2005). Evaluation of Dietary Natustat for Control of Histomonas meleagridis in Male Turkeys on Infected Litter. Avian Diseases, vol. 49, issue 3, pp. 423-5. 
[13] Duskaev, G. K., et al. (2018). Effect of the Combined Action of Quercus Cortex Extract and Probiotic Substances on the Immunity and Productivity of Broiler Chickens Veterinary World, vol. 11, issue 10, pp. 1416-22.

[14] Fisinin, V. I., et al. (2018). Mixtures of Biologically Active Substances of Oak Bark Extracts Change Immunological and Productive Indicators of Broilers. Sel'skokhozyaistvennaya Biologiya, vol. 53, issue 2, pp. 385-92.

[15] Xu, J., et al. (2018). Epidemic Characteristics of Clinical Histomoniasis in Chicken Flocks in Eastern China. Avian Diseases, vol. 62, issue 2, pp. 189-94.

[16] Hauck, R., et al. (2018). Retrospective Study of Histomoniasis (Blackhead) in California Turkey Flocks, 2000-2014. Avian Diseases, vol. 62, issue 1, pp. 94-100.

[17] Hauck, R. and Hafez, H. M. (2013). Experimental Infections with the Protozoan Parasite Histomonas Meleagridis: A Review. Parasitology Research, vol. 112, pp. 19-34.

[18] Van der Heijden, H. M., et al. (2006). Genotyping of Histomonas Meleagridis Isolates based on Internal Transcribed Spacer-1 Sequences. Avian Pathology, vol. 35, pp. 330-4. 